EDUCATION AND TRAINING

\title{
Clinicians, librarians and patient safety: opportunities for partnership
}

\section{Zipperer}

Qual Saf Health Care 2004;13:218-222. doi: 10.1136/qshc.2002.003103

Librarians could improve the safety of medical care through greater participation in patient safety initiatives. A librarian's expertise in accessing the evidence base could enhance the safety and appropriateness of care in a clinical environment. In addition, librarians could apply specific technical knowledge management skills to medicine. To realize improvements from these skill sets, healthcare leaders must consider ways of working with librarians to enhance patient safety.

"If only we could have two lives: the first in which to make one's mistakes, which seem as if they have to be made; and the second in which to profit by them." D H Lawrence

( ne major factor in improving and maintaining patient safety is timely access to appropriate patient information, medical evidence, and relevant institutional knowledge. However, the range of what constitutes complete information in patient safety has yet to be defined by the multidisciplinary teams involved in safety. A reciprocal commitment to engage librarians in safety programs is likely to benefit clinicians and patients alike through improved knowledge and information management in the healthcare environment. Now is an optimal time for such collaboration, given the demands that clinicians face each day in using information in an increasingly time constrained environment.

Research or organizational scientists have yet to identify and promote the role that librarians can apply to safety improvement in medicine. To the author's knowledge, no "call for papers" to engage individuals in new areas of research, requests for proposals, or conference tracks have been dedicated to exploring the value of this partnership. This discussion challenges the patient safety community to make a commitment to explore this issue. Examples of how the skills of librarians can be applied to affect care in

Correspondence to: L Zipperer, Zipperer Project Management, 1002 Washington Street \#3E, Evanston, IL 60202, USA; lorri@zpml.com

Accepted for publication 29 November 2003 patients may well have been the 2001 incident in the United States at the Johns Hopkins Health System, Baltimore, Maryland (box 1). ${ }^{12}$ In this tragic instance a literature review was not effectively completed for a non-FDA approved use of a medication to be used in a human subjects trial. The ingestion of the drug hexamethonium was documented in pre-1966 medical literature to have adverse effects. Unfortunately, this literature base was not searched by the research team. The pre-1966 literature was not searchable at the time via the web based version of the US National Library of Medicine's PubMed database. Articles describing the risks of hexamethonium appeared in the index before 1966 which a librarian who undertook a rigorous search of the literature would probably have found. In this instance, they were not found through a conventional search of the database. This misstep is just one of several that played a part in the complexities that resulted in the loss of life of a healthy volunteer. ${ }^{3}$

Clinical trials are complex undertakings that are rife with the potential for errors of omission and commission. However, it is possible that, if a member of the librarianship profession had participated in or been mentioned at conferences or educational events dedicated to patient safety, this mistake may have been avoided due to an increased recognition by clinicians of the value of librarians as key participants in increasing medical safety.

\section{SKILLS IMPACTING ON CARE}

Primary studies have shown that the expertise of librarians can make a difference in clinical care by locating materials that remind practitioners of facts or details, support various courses of diagnostic or therapeutic action, or provide new pieces of information that modify or redirect clinical activities. ${ }^{45}$

Through the advent of the Internet and the free availability of MEDLINE, access to the medical literature has changed dramatically, as has the interaction with librarians as the professional navigators of that domain. Clinicians do more of their own research if time, access, information seeking skills, and interest permit. This is not often the case. They still seek ways to manage the information needed to address the clinical questions for which they do not have ready answers. ${ }^{6}$ The relationship between librarians and clinicians should therefore be strengthened to facilitate effective work processes and improve patient safety through access to better evidence and answers. 


\section{Box 1 Johns Hopkins incident}

In June 2001 a 24 year old female volunteer died in a research study at the US based Johns Hopkins University Asthma and Allergy Center in Baltimore, Maryland. The patient was recruited as a normal volunteer in a study of asthma funded by the US National Institutes of Health. The purpose of the study was to understand how bronchodilation is maintained in healthy patients by deep inspirations in the face of a bronchoconstrictive stimulus, and the specific physiology of the process.

The volunteer began her involvement in the research in April 2001. She participated as outlined in the IRB approved protocol. As articulated by the protocol, she received approximately $1 \mathrm{~g}$ hexamethonium by inhalation to produce symptoms similar to those of an asthma attack. Soon afterwards she exhibited a dry cough and flu-like symptoms. The following week additional pulmonary stress was experienced and reductions in her arterial oxygen saturation after minimal exertion were noted. She was admitted to The Johns Hopkins Bayview Medical Center (JHBMC) in Maryland for observation where her condition worsened, culminating in adult respiratory distress syndrome (ARDS) and kidney failure. She died 2 weeks later.

Officials at Johns Hopkins accepted responsibility for the incident and set to work to determine the exact cause of what happened by calling for an in-depth internal investigation. The full report of the investigation has been made widely available on the internet at http://www.hopkinsmedicine.org/ researchvolunteerdeath.html. No distinct cause for the death was identified although weaknesses in the process were noted.

Among the lapses cited in the report was the incomplete literature review for the non-FDA approved use of hexamethonium. The committee noted that, while the primary investigator had made a good faith effort to fully review the applicable literature, more evidence describing the risks of inhaled hexamethonium were available and, if identified, located and reviewed, may have alerted the research team to the possible dangers. It was felt by the committee that the Internal Review Committee for the research should have been more rigorous in its requirement for examination of the evidence.

In the initial wake of the incident, Johns Hopkins had its US government funding briefly suspended. It was reinstated on 22 July 2001.

\section{ORGANIZATIONAL ACTIONS}

The importance of information delivery as a tool to enhance patient safety conflicts with the anecdotal evidence of declining funding for medical libraries and the professionals who run them. For example, in the USA the Joint Commission on Accreditation of Healthcare Organizations (JCAHO) used to require the operation of a functional library within a hospital. Language was changed to reflect a certain level of information readiness and preparedness, but not to indicate that the librarian was a key player in leading that effort. The requirement for a "library" was removed in 1994. Although information management is still considered to be vital-as reflected in the accreditation standard that calls for effective information management and access to knowledge based information ${ }^{7-10}$ - anecdotal evidence suggests that the lack of a specific requirement for a library in a hospital has resulted in the closure of many libraries (Gluck, personal communication). Research should be undertaken to explore the impact of this change on safety as the existing evidence is primarily subjective.
Librarians continue to be concerned that, without that mandate from JCAHO, their funding may be seriously reduced, hampering their ability to participate in patient safety and quality improvement efforts. As with any strategic undertaking, clear and unambiguous messages aid management in garnering support for activities that are resource intensive such as information efforts inside and outside the traditional library environment. A specific connection between the skills of the librarian and patient safety may help to bolster justification for the presence of a librarian in a clinical environment. Some specific organizational activities have taken place to aid in establishing such a relationship. These include:

- The National Patient Safety Foundation (NPSF): founded in Chicago in 1997 with a librarian on its staff. The NPSF recognized that to address its education and communication goals, strategic information and knowledge needed to play a key role in its activities and that a professional should be retained to do the work. Information tools to inform the patient safety community such as a multidisciplinary bibliography and an email discussion group came out of that partnership. ${ }^{11}$

- The US based Medical Library Association (MLA): this has represented the health sciences information professional community for over 100 years. In 2002 a revised set of Standards for Hospital Librarians was developed with language that links the hospital librarian with patient safety programs. ${ }^{12}$

- In light of the Johns Hopkins incident, MLA developed guidelines to promote best practices involving expert literature searching in health care and biomedical research institutions. ${ }^{13}$

In an effort to educate beyond the medical library community, the Special Library Association-a US based organization of 15000 international members representing corporate information centers, private libraries and libraries that serve a diverse set of distinct audiences-provided a mechanism for education. It presented two conference sessions (one in 1999 and one in 2001) that were designed to highlight the need for collaboration between librarians and healthcare leaders and clinicians to improve sharing of safety information. ${ }^{14} 15$

\section{CHANGING PARADIGMS}

Safety experts are exploring how a lack of teamwork and partnering affects the care of patients. Nurses, pharmacists, physicians, administrators, and risk managers have widely acknowledged responsibilities. Similar clear and forceful articulation of a librarian's role will support their involvement in the patient safety team. This role should improve patient safety through effective access to information and knowledge for clinical, research, and organizational efforts. Physicians and administrators are in the best position to articulate and support this role. The library community is beginning to embrace the chance to become involved in this way. The informationist concept, evidence based medicine, and knowledge management provide such opportunities.

\section{The informationist}

A dialogue was launched in 2000 that called for a paradigm shift in how clinicians and librarians thought about their intersecting roles in medical care. Davidoff and Florance, ${ }^{16}$ in an editorial in the Annals of Internal Medicine, outlined elements of a structured clinical role for librarians called an 
informationist, an extension of the concept of a clinical librarian. ${ }^{17}$ The programs supporting this responsibility would require librarians to acquire clinical knowledge; expertise in the retrieval, synthesis, and provision of information; attend accredited training programs; and be actively involved in the process of care. Health sciences information professionals are still discussing the implications of the movement and continue to challenge the status quo. ${ }^{18}$ This new paradigm is prompting the pursuit of a more clearly defined role for librarians in clinical care that could affect patient safety. ${ }^{19}$ Organizational and monetary support for these programs should be championed by physicians and administrators.

\section{Evidence based medicine}

Evidence based medicine (EBM) is the conscientious, explicit and judicious use of current best evidence in making decisions about the care of individual patients. The practice of EBM means integrating individual clinical expertise with the best available external clinical evidence from systematic research. ${ }^{20}$ This process can influence safety in two ways. Firstly, it supports the expanding knowledge base that facilitates safety research. EBM efforts to collect and analyze the literature related to safety have been undertaken in both the US and UK, ${ }^{21}{ }^{22}$ but experts have also noted that efforts to improve safety should not necessarily be limited to those things that had been studied in depth and for which extensive hard evidence was available. ${ }^{23}$ This indicates a need for a multifaceted approach to knowledge collection that librarians are uniquely positioned to undertake.

Secondly, EBM can affect safety by providing a direct link between the medical literature and patient care. As the medical information base expands and the pressure on clinicians' time increases, the ability of librarians to find evidence to address distinct clinical problems becomes more essential. The safety implications of access to this information should be emphasized to support the training and collaboration required to build and sustain productive relationships as a regular course of providing care. Studies exploring the outcomes of health library services have been undertaken in a number of countries including the US, Spain, Australia and New Zealand. ${ }^{24}$ In a seminal 1992 study based in the US, Marshall found that $94 \%$ of physicians surveyed assessed that information from the library contributed to higher quality of care. ${ }^{25}$ This strong level of support should generate efforts to involve more medical librarians in this fashion. However, Marshall acknowledges that this study was subjective and that more concrete work needs to be undertaken to quantify the impact of librarians on clinical care. A good example is the research currently underway at the US based Vanderbilt Medical Center's Eskind Biomedical Library by Giuse to evaluate the success of their clinical informationist program.

Librarians have not only become active participants in providing research based on EBM principles but are currently providing training in quality filtering, critical analysis of literature, and targeted searching techniques to a variety of audiences including medical students and other clinicians. ${ }^{26}$ By improving clinicians' understanding of the evidence and how best to locate it, librarians will help increase its application to safe patient care.

\section{Knowledge management}

There are a number of definitions of knowledge management, but the one used here is that by Dalrymple ${ }^{27}$ which states that "knowledge management enhances the use of organizational knowledge through sound practices of information management and organizational learning". The learning organization concepts of discussing mistakes openly to learn from them, building trust, nurturing teamwork, and encouraging management interaction with employees are key to the development of a culture of safety. Not only have studies found that information provided by librarians modified both current and future clinical activities, but also that librarians can spur organizational growth through the ability to share history, support project groups, and translate stories for others to learn. ${ }^{28}$ Librarians often work with a representative set of users within their environments and are thus well positioned to facilitate a culture of learning. Through this interaction with clinicians, administrators, students and patients, librarians can build bridges between those at the "sharp end" (at the point of care) and those at the "blunt end" (the administrators). ${ }^{29}$

Efforts to share knowledge in hospitals create opportunities for teamwork with librarians. Their skills prepare them to contribute to projects as developers of access points to the literature, selectors of the databases and web sites to enhance knowledge management data systems, and organizers of the taxonomies to locate the stories and records within the data systems. In addition, they can work with system designers to help make the knowledge management system an effective story capturing and comparison tool.

Librarians are participating in the creation of information and knowledge tools at the bedside. Partnerships such as that of the health sciences librarians at the University of Washington provide an example of such a partnership. The librarians took part in the web based patient medical record system project as facilitators, publishers, integrators, and educators to develop a clinical information tool to support knowledge based care. ${ }^{30}$

Knowledge management skills can also be useful in nonclinical medicine. They can support organizational learning and innovation through the development of tools to capture and share in-house "lessons learned". Librarians from nonmedical environments-targeted by leading knowledge management authorities Davenport and Prusak as "indispensable knowledge brokers" ${ }^{\prime \prime 1}$ - have implemented knowledge management programs in business and could contribute in unique ways to achieve initial successes for medicine if given the opportunity.

\section{PARTNERING FOR SAFETY \\ Efforts at the local level}

Health care has embraced the need to include non-clinical staff in order to establish a robust culture of safety. The above review suggests that information based contributions to safety can best be envisaged through partnerships. Librarians, hospital administrators, and clinicians can collaborate at the local level through the following actions:

- Incorporating information and knowledge management goals specifically in the strategic plans of safety efforts.

- Inviting librarians to the table to help make change: team involvement of librarians will change global information and knowledge management in medicine.

- Encouraging the reporting of ineffective literature searching as a "near miss" to enable organizations to learn about circumstances where opportunities for errors related to this gap in effective practice can affect care.

- Identifying a tendency to ignore literature review for care as a "risky behavior" that needs to be modified to improve safety. Practitioners will not only improve their competence for searching but will know when they are not successful in the work they do, and will seek out medical librarians as the experts on their team to enhance their clinical decision making process.

- Encouraging and enabling librarians to participate in clinical activities such as executive walkarounds, grand 
rounds, morning reports, institutional review boards, and mortality and morbidity conferences.

- Supporting informationists and/or other clinical roles outlined by the librarians to use their expertise effectively in the clinical setting. Some examples include: working with the pharmacy to populate new online formularies with the appropriate drug information and providing information for patients on their condition so they can be their own safety advocates. ${ }^{32}$

- Establishing a concrete role for librarians in the research needed to ensure safe development of clinical trial protocols. ${ }^{33}$ This is being implemented on an ad hoc basis and should be added to the "best practices" canon for patient safety.

- Marketing information access skills to ensure that clinicians are aware of the role of library services in safety and research. Librarians should work toward shaping an outcome based case to present to administration and practitioners on the importance of information management in safe patient care. This is another opportunity for librarians to partner with pharmacists who are trained in searching the pharmacology and pharmacy literature and often provide information from that resource base at the clinical level.

- Enhancing the need for patient safety knowledge to be expected reading in medical environments. The addition of several patient safety, quality improvement titles to the Brandon Hill list-the regularly updated suggested titles list for hospital and small medical libraries that provides authoritative guidance to book purchases-would assist in spreading this message. ${ }^{34}$

- Developing and implementing evaluation methods that provide tools to document the efficacy and effectiveness of librarians' involvement in safety efforts.

\section{Achieving global change}

Global change can begin to take place only through awareness. The librarian and medical communities can affect change by working together as partners. Through this partnership, innovative research and knowledge sharing strategies will result that may lead to safer care. Efforts that have the potential for global impact include:

- Providing and/or seeking opportunities for cross fertilization of the ideas and expertise of librarians, their champions, and others interested in the issue. Establishing venues for writing about what they do and know in the peer reviewed literature.

- Promoting conference programmes for a variety of audiences (librarians, information technologists, healthcare management and leadership, and patient safety specialists) that focus on building teamwork among healthcare information, clinical, and administrative personnel.

- Developing crossover curricula for clinicians and librarians who wish to combine their skills to become informationists or clinical librarians. Academic medical centers affiliated with library sciences and information studies programs are aptly positioned to implement this change. In October 2003 the US National Institutes of Health launched a National Library of Medicine fellowship program to provide funding for individuals to provide education across disciplines.

- Exploring, assessing, and illustrating the risks involved in not getting the best information prior to and while providing patient care. This provides an opportunity for illustrating how errors can occur through the use of
Key messages

- A commitment to involve librarians in patient safety initiatives could improve the safety of medical care.

- Organizational efforts are beginning to appear that support the role of librarians in patient safety initiatives.

- Individuals and hospitals can amend working relationships to enrich the role of librarians in safety programs.

- Universal efforts and explorations into the relationship between librarians and patient safety initiatives should be undertaken.

established process assessment techniques such as failure mode and effects analysis (FMEA).

- Creating a robust taxonomy controlled vocabulary/thesaurus that can be used effectively to manage the literature required for the study of patient safety. MESH, the vocabulary supporting MEDLINE, has served its initial purpose admirably in this regard, but its terminology is not sufficiently detailed nor is the scope of the corresponding database broad enough to represent fully the intricacies of the patient safety knowledge base. ${ }^{35}$ This is a government level challenge. The National Library of Medicine and the Agency for Healthcare Research and Quality, both arms of the US Department of Health and Human Services, could lead in this effort through funding or manpower initiatives.

- Building or funding a consortium of libraries representing specialized collections that provide access to the multidisciplinary knowledge needed by researchers from materials that are hard to find so that they can fully explore and understand safety issues.

- Spearheading an effort to enhance evaluation skills and tools for documenting the efficacy and effectiveness of these global change methods and, ultimately, the efficacy and effectiveness of librarians in improving patient safety. For example, at present there is no information on the performance of hospitals with or without libraries. ${ }^{36}$

\section{CONCLUSIONS}

Participants in all fields and at all levels of health care should seek out and embrace possibilities to encourage effective working relationships that enhance safety. Librarians can contribute to safety in a variety of ways, given the appropriate conditions for their involvement. These efforts should be validated to underscore their value and efficacy. The suggested methods of local partnering and global change projects offer additional avenues to foster improvement for clinical organizations and their staffs. These suggestions, coupled with the professional motivation of many in the healthcare and library communities, could foster the research, creative ideas, and partnerships needed to involve the information profession more completely in improving patient safety.

\section{ACKNOWLEDGEMENTS}

The author thanks Jill Blim, Frank Davidoff, Jeannine Gluck, Ruth Holst, Diane Hummel, Cathy Tokarski, and Diane Wolf for their time and comments in shaping this paper.

Lorri Zipperer is a librarian and works as a consultant on patient safety information projects. She served as the Information Project Manager for the National Patient Safety Foundation from 1996 to 2000. 


\section{REFERENCES}

1 McLellan F. 1966 and all that-when is a literature search done? Lancet 2001;358:646

2 Perkins E. Johns Hopkins' tragedy: could librarians have prevented a death? Medford, NJ: Information Today, 7 August 2001. http:// www infotoday com/newsbreaks/nb010806-1 htm.

3 Anon. Johns Hopkins medicine research volunteer death. Baltimore: Johns Hopkins University \& Johns Hopkins Health System, 2001. http:// www.hopkinsmedicine.org/researchvolunteerdeath.html.

4 Schacher LF. Clinical librarianship: its value in medical care. Ann Intern Med 2001;134:717-20.

$5 \mathbf{O}^{\prime}$ Connor P. Determining the impact of health library services on patient care: a review of the literature. Health Info Libr J 2002;19:1-13.

6 Ely JW, Osheroff JA, Ebell MH, et al. Obstacles to answering doctors' questions about patient care with evidence: qualitative study. BMJ 2002;321:1-7.

7 Rand DC, Gluck JC. Proactive roles for librarians in the JCAHO accreditation process. J Hosp Librarianship 2001; 1:25-40.

8 Plutchak TS. Determining value. J Med Libr Assoc 2002;90:273-5.

9 Joint Commission on Accreditation of Healthcare Organizations. Information management planning. Hospital accreditation standards. 2004 prepublication edition. Standard 1M1.10. Oakbrook, IL: Joint Commission on Accreditation of Healthcare Organizations (JCAHO), 2003. http:// www.jcaho.org/accredited+organizations/2004+standards.htm.

10 Joint Commission on Accreditation of Healthcare Organizations. Knowledge based planning. Hospital accreditation standards. 2004 pre-publication edition. Standard 1M.50.10. Oakbrook, IL: Joint Commission on Accreditation of Healthcare Organizations (JCAHO), 2003. hittp:// www.jcaho.org/accredited+organizations/2004+standards.htm.

11 National Patient Safety Foundation. www.npsf.org.

12 Gluck CR, Hassig RA, Balogh L, et al. for the Standards Committee, Hospital Librarians Section, Medical Library Association. Standards for hospital libraries 2002. J Med Libr Assoc 2002;90:465-72.

13 Medical Library Association. Medical Library Association policy statement: role of expert searching in health sciences libraries. 2003. http:// www.mlanet.org/pdf/expert_search/policy_expert_search.pdf.

14 Funk C, Hatlie M. Role of information in the quality of health care. Presentation at SLA/Biomedical and Life Sciences Division. SLA 90th Annual Conference Minneapolis, MN, 1999.

15 Dalrymple PW, Schuve P. Knowledge management and medicine: building bridges for better patient care. SLA/Biomedical and Life Sciences Division. Presentation at SLA 92th Annual Conference, San Antonio, TX, 2001

16 Davidoff F, Florance V. The informationist: a new health profession? Ann Intern Med 2000;132:996-8.

17 Jerome RN, Giuse NB, Gish KW, et al. Information needs of clinical teams: analysis of questions received by the Clinical Information Consult Service. Bull Med Libr Assoc 2001;89:177-84

18 Shearer BS, Seymour A, Capititani C, eds. Ptient centered librarianship: the informationist and beyond. A symposium to honor the 50th anniversary of the Philadelphia Regional Chapter of the Medical Library Association. J Med Libr Assoc 2002;90:21-85.
19 Shipman JP, Cunningham DJ, Holst R, et al. The informationist conference: report. J Med Libr Assoc 2002;90:458-64.

20 Sackett DL, Richardson WS, Rosenberg W, et al. Evidence-based medicine how to practice and teach evidence-based medicine. New York: Churchill Livingstone, 1997:2.

21 Shojania KG, Duncan BW, McDonald KM, et al. Safe but sound: patient safety meets evidence-based medicine. JAMA 2002;288:508-13.

22 Westwood M, Rodgers M, Sowden A. Patient safety: a mapping of the research literature. NHS Centre for Reviews and Dissemination. Patient Safety Research Programme, University of Birmingham, UK. http:// www.publichealth.bham.ac.uk/psrp/pdf/ Mapping\%20of\%20the\%20Research\%20Literature\%20-\%20Sowden.pdf.

23 Leape LL, Berwick DM, Bates DW. What practices will most improve safety? Evidence-based medicine meets patient safety. JAMA 2002;288:501-7

$24 \mathbf{O}^{\prime}$ Connor $\mathbf{P}$. Determining the impact of health library services on patient care: a review of the literature. Health Info Libr J 2002;19:1-13.

25 Marshall JG. The impact of the hospital library on clinical decision making: the Rochester study. Bull Med Libr Assoc 1992;80:169-78.

26 Scherrer CS, Dorsch JL. The evolving role of the librarian in evidence-based medicine. Bull Med Libr Assoc 1999:87:322-8.

27 Dalrymple PW. Knowledge management in the health sciences. In: Kanti Skirantaiah T, Koenig M, eds. Knowledge management for the information professional. Information Today Inc, 2000:389.

$28 \mathbf{O}^{\prime}$ Connor P. Determining the impact of health library services on patient care: a review of the literature. Health Info Libr J 2002;19:1-13.

29 Zipperer LA, Gluck J, Anderson S. Knowledge maps and patient safety. J Hosp Librarianship 2002;2:17-36.

30 Fuller SS, Ketchell DS, Tarczy-Horwoch P, et al. Integrating knowledge resources at the point of care: opportunities for librarians. Bull Med Libr Assoc 1999;87:393-403.

31 Davenport TH, Prusak L. Working knowledge. Boston: Harvard Business School Press, 2000:29.

32 Holman JM. The role of medical librarians in reducing medical errors. HealthLeaders Online. 16 September 2002. http://www.healthleaders.com/ news/print. php? contentid $=38058$.

33 Resnick R. IRB responsibilities - comments. Posting on Medical Libraries Discussion List. 30 August 2001. http://listserv.acsu.buffalo.edu/cgi-bin/ wa? $\mathrm{A} 2=$ ind $0108 \mathrm{e} \& \mathrm{~L}=$ medlib- $\& \mathrm{~T}=0 \& \mathrm{~F}=\& \mathrm{~S}=\& \mathrm{P}=3874$.

34 Hill DR, Stickell HN. Brandon/Hill selected list of print books and journals for the small medical library. Bull Med Libr Assoc 2001;89:131-53.

35 Westwood M, Rodgers M, Sowden A. Patient safety: a mapping of the research literature. NHS Centre for Reviews and Dissemination. Patient Safety Research Programme. University of Birmingham, UK. http:// www.publichealth.bham.ac.uk/psrp/pdf/ Mapping\%20of\%20the\%20Research\%20Literature\%20-\%20Sowden.pdf.

36 Adams H. Performance of hospitals/physicians without libraries. Posting on Medical Libraries Discussion List. 14 January 2003. http:// listserv.acsu.buffalo.edu/cgi-bin/wa?A2 = ind0301 B\&L = medlib$|\& P=R 4817 \&|=-3$. 